\title{
Development and Application of Tool Coatings*
}

\author{
Guo Yuyao ${ }^{1}$, Meng Deqiang ${ }^{1}$,Lin We ${ }^{1}$,Hou Xiang ${ }^{1}$,Tang Kuanyu ${ }^{1}$, \\ ${ }^{1}$ Tianjin Key Laboratory of High Speed Cutting and Precision Manufacturing, \\ Tianjin University of Technology and Education \\ Tianjin, China
}

\begin{abstract}
With the rapid development of modern manufacturing, traditional tools can no longer meet the needs of current processing. The appearance of coated tools has greatly solved this problem. Compared with traditional tools, coated tools have excellent thermal stability, good wear resistance, high hardness and high bonding strength. This article mainly introduces the development of the coating material of the tool, the preparation technology of the coating and the prospect of the application and development of the tool coating.
\end{abstract}

Keywords: Tool Coating; Coating Material; Preparation Technology; Application Prospect And Development

\section{INTRODUCTION}

With the continuous development of processing technology in the machinery industry, the use of new materials with higher processing difficulty has gradually increased, and the conditions for high-speed cutting continue to increase. Traditional tools can no longer meet new requirements[1]. Although through a series of measures, the hardness of the tool material is increased and the wear resistance is increased, at the same time, the impact toughness and the bending strength of the tool material are also reduced, so the performance of the tool is affected. Because the tool coating is an effective method to improve the above problem, the tool substrate is coated with one or more layers of high hardness, wear-resistant, corrosion-resistant metal or non-metallic coating (eg: $\mathrm{Cr}-\mathrm{N}$, Al-Ti-N, Al-Cr-N, Al2O3 Such as), which makes the friction factor between the tool and the work-piece greatly reduced, without increasing the hardness and toughness of the coating under the premise of improving the wear resistance of the tool, extending the service life of the tool and improving the processing efficiency [2] .

\section{DEVELOPMENT OF TOOL COATING MATERIALS}

In recent years, the progressive development of the tool coating has made it possible to increase the coating selectivity. For example, binary coatings, multi-component coatings, nano-composite coatings, and alternately coated multi-element coatings. The following is the development and application of some tool coating materials and preparation processes. Which kind of coating is used in different processing conditions and different processing occasions is beneficial to the cutting process to obtain the best effect. The characteristics of each kind of coating material have a very important role.

\section{A. Binary coating}

The transition metal nitride hard coating prepared by plasma-assisted physical vapor deposition (PVD) technology can be widely used to improve the performance and life of industrial cutting tools and mechanical parts. Among them, the binary coating $\mathrm{CrN}$ is widely used in the machining industry as a protective coating because of its high hardness, strong film/base bond strength, and wear resistance. The high oxidation temperature and excellent corrosion resistance under harsh environmental conditions have further improved the overall performance of $\mathrm{CrN}$ coatings and have been cooperating with other metals to form multi-component coatings[3]. TiC is a high hardness, wear resistant compound II coating with good anti-friction and abrasion resistance; $\mathrm{TiN}$ is slightly lower in hardness but chemically stable and reduces the friction between the tool and the work-piece being machined. Coefficient, combined with high-speed steel coating firmly. From the process point of view, both are ideal coated tool materials, but the binary coating is difficult to meet the current requirements of high-speed cutting and harsh processing conditions on the coating performance of the tool [4].

\section{B. Three yuan coating}

The addition of aluminum to the binary coating $\mathrm{CrN}$ increases the hardness, wear resistance and high temperature oxidation resistance significantly, and as the aluminum content increases, the oxidation rate of the ternary CrAlN coating decreases and the oxidation resistance increases. TiAlN coating has higher anti-oxidation temperature than $\mathrm{TiC}$ and TiN. Under high temperature conditions, TiAlN has higher hardness than TiN coating, low thermal conductivity, and strong heat insulation effect. It is suitable for dry machining and other harsh processing conditions[5]. However, compared with TiAlN coatings, CrAlN coatings have a greater potential for improving oxidation and wear resistance based on coatings. Therefore, many recent studies have focused on aluminum-rich AlCrN PVD coatings. Nano-composite $\mathrm{CrSiN}$ coating has both anti-adhesion and wear resistance, CS coating nano-crystalline particles, or a silicon-rich non-crystalline composite. Therefore, it has good lubricity and hardness. It can be used to solve the problem of sticking and detachment of built-up edge and reduce the surface roughness of the work-piece and increase the service life of the tool. These coatings are particularly useful for tools and mechanical components. Other tribological protection coating applications [6]. 
With the application of new materials in the mechanical processing industry, the development of coatings can further solve the difficult problems of difficult-to-cut and difficult-to-machine materials. The emergence and development of multi-layer coatings, nano-composite coatings, functional gradient coatings, etc., due to the combination of the excellent characteristics of various materials, occupies a large position in the current tool coating.

\section{Nano-composite coating}

As a new generation of film materials, nanocomposite films can effectively improve the toughness of thin films, the nanocomposite structural film were formed by isolated nanocrystals embedded in a very thin amorphous layer, the nano-crystalline has higher hardness, the amorphous phase has better plasticity, the interfacial energy of the two phases is high, and the crystalline phase and the amorphous phase are thermodynamically separated; In addition, Dislocations cannot be formed in tiny nanocrystals, the thin amorphous layer that between the grains can effectively prevent the grain boundary from slipping, and a large number of two-phase interfaces increase the micro crack propagation resistance. Therefore, this film has a high hardness (>40 GPa), high toughness and excellent wear resistance, suitable for high speed cutting, dry machining and other conditions[7,8].

\section{Multi-layer composite coating}

The multi-layer composite coating is obtained by changing the structure and chemical composition. It is generally formed by alternately stacking two different materials at a certain period, and the thickness of each layer can be controlled to be several nanometers or less. Make full use of the advantages of each material, and more importantly, synergistic effects such as super modulus effect, super hard effect, etc.[9]. The multi-layer composite coating can further improve the toughness, hardness and high temperature oxidation resistance of the single coating based on the original properties of the single coating to better meet the high-efficiency processing of modern difficult-to-machine materials[10,11]. In production applications, Oerlikon Balzas Coatings has applied $\mathrm{AlCrO} / \mathrm{AlCrN}$ multi-layer composite coatings to gear hob surfaces and has accounted for the tool coating industry, There is an absolute advantage.

\section{COATING PREPARATION TECHNIQUES}

Vacuum vapor deposition of a tool coating is formed by vacuuming a metal or metal oxide into a gaseous atom or molecule by various methods and depositing it on a metal or non-metal surface. Compared with the traditional electroplating method, vacuum coating has the advantages of low energy consumption, non-toxicity, no waste liquid, low pollution, low cost, good decorative effect and strong metal feeling. It is a promising new technology. Vacuum is one of the necessary conditions for vapor deposition of coating. Under vacuum, the active reactant gas has low oxygen content, and has the characteristics of less collision between gas molecules, chemical inactivity, low thermal conductivity and low pressure. The porosity and impurity content of the film are low; the degree of vacuum also affects the kinetic energy of the deposited ions, changes the growth mechanism of the coating, and refines the grains, affecting the phase structure and distribution of the coating, thereby obtaining a coating of high hardness and high purity. Vapor deposition is a method of forming a metal or compound coating with special properties on the surface of an object by utilizing physical and chemical reaction processes occurring in a vacuum gas phase. The basic principles of vapor deposition techniques according to coating formation include: chemical vapor deposition (CVD) [12]; physical vapor deposition (PVD) [13].

PVD: it is a process of condensing coating in vacuum environment by means of physical vapor phase such as sputtering or evaporation. At present, the main methods of physical vapor deposition include sputtering coating, vacuum evaporation coating, arc, hollow cathode, reactive plasma coating. The deposition process is carried out in vacuum and gas discharge (low pressure plasma), high current arc discharge and low voltage. The reaction source material of the coating is solid material. After "evaporation or sputtering", the reacting gas through "ionization, recombination and reactant deposition" has been formed on the surface of the parts to produce a new solid substance and deposited on the surface of the workpiece to form a uniform and uniform hard coating and their composite coating. With the rapid development of high and new technology, new bright spots have appeared in physical vapor deposition, such as multi arc ion plating and magnetron sputtering compatible technology, unbalanced magnetron sputtering and twin target technology, which make physical gas phase deposition continuously meet the development of big industrial model.

CVD: the process technology of solid film or coating made by chemical reaction under a certain temperature and gaseous condition by plasma excitation and heating. Chemical vapor deposition (CVD) is a very flexible and widely used chemical vapor growth method for preparing coatings. It is a method of forming a required solid film or coating on the surface of the matrix by supplying a single or multiple compound containing a thin film element to a reaction chamber which is provided with a matrix. The activation energy can also be obtained by plasma and laser radiation technology, so that it can be deposited at lower temperature. Now chemical vapor deposition (CVD) is not only widely used in the deposition of a variety of single crystal, polycrystalline or glass inorganic membrane materials, but has become a new field of inorganic synthetic chemistry.

\section{PROSPECT OF RESEARCH ON HARD COATING MATERIALS}

As a coating material applied to a tool, whether it can extend the service life of the tool is always the most urgent problem to be solved in the machining industry. This requires the continuous development of the coating material and its current status through research and understanding. A well-prepared and well-designed coating process is used to prepare a tool coating with excellent overall performance. In summary, it can 
be seen that the higher hardness alone does not improve the overall performance of the coating. Therefore, important applications include whether it has a hard coating with a low coefficient of friction and self-lubricating properties. This has led to the development of coating materials in the direction of diversification, gradients, and multilayers. The preparation process and latest development status of the tool coating materials can provide theoretical support and certain reference value for the application and research of coating materials.

\section{CONCLUSION}

The application of the coated tool has effectively solved the rigidity of the tool and solved the contradiction between the inability to coexist between the rigidity and the toughness of the tool, and greatly enhanced the tool life and impact resistance to a certain extent. However, the problem of poor toughness and impact resistance of the tool itself has not been fundamentally solved.The cost of processing is high. Therefore, in order to improve the overall performance of coated tools, hard cutting, dry cutting and high speed cutting should be vigorously developed.

\section{REFERENCES}

[1] Han Min jian. The development and research of coating technology of tool surface[J], Journal of Lanzhou Polytechnic College,2010, 17(3): 48-52

[2] Xiang Si qin. Present situation and development of tool coating materials $[\mathrm{J}]$, Prospect of science and technology,2016, 26(28): 296

[3] Ferreira F, Oliveira J C, Cavaleiro, et al. CrN thin films deposited by HiPIMS in DOMS mode [J]. Surface \& Coatings Technology, 2016, 291: 365-375

[4] Chen Wei xi. Developing Status and Views for Cutting Tool Coating Technology[J], Tool technology,2000, 34(3): 3-5

[5] Mo J L, Zhu M H, Leyland A, et al. CrN thin films deposited by HiPIMS in DOMS mode[J]. Surface \& Coatings Technology, 2016, 291: 365-375

[6] Yuan Jia dong. Nteractive development of coating technology and modern cutting tools[J], Surface modification technology, 2007, 32(8): 1-4

[7] Vepřek S, Zhang R F, Vepřek-Heijman M G J, et al. Superhard nanocomposites: Origin of hardness enhancement, properties and applications[J]. Surface \& Coatings Technology, 2010, 204: 1898-1906.

[8] Lawal J, Kiryukhantsev-Korneev P, Matthews A, et al. Mechanical properties and abrasive wear behaviour of Al-based PVD amorphous/nanostructured coatings[J]. Surface \& Coatings Technology, 2017, 310: 59-69.

[9] Zhao Wen ji, Kong Ming, Wu Xiao yan, et al. Study on the sensibility of hardness of TiN/Si3N4 nano-multilayers to $\mathrm{Si}_{3} \mathrm{~N}_{4}$ layer thickness[J]. Acta Metallurgica Sinica, 2007, 43(2): 154-158.

[10] Zhou Guang hong, Fang Dong, Yin Feng, et al. Synthesis and characterization of bionic Ti/TiB2 Multilayer[J]. Chinese Journal of Vacuum Science and Technology, 2015, 35(7): 819-824.

[11] Li T S, Li H, Pan F. Microstructure and nanoindentation hardness of Ti/TiN multilayered films[J]. Surface and Coatings
Technology, 2001, 137( 2) : 225-229.

[12] Wei Q P, Yu Z M, Ashfold M N R, et al. Effects of thickness and cycle parameters on fretting wear behavior of CVD diamond coatings on steel substrates [J]. Surface \& CoatingsTechnology, 2010, 205 (1): 158-167.205 (1): 158-167.

[13] Strnad G, Buhagiar J. Lastest developments in PVD Coatings for Tooling [J]. Scientific Bulletin of the "Petru Major" University of Targu Mures, 2010, 7 (XXIV) (1): 32-37 